\title{
APLICATIVO PARA ENSINO DE ENGENHARIA QUÍMICA BÁSICA
}

\author{
J. C. PERISSÉ 1 , A.VALDMAN ${ }^{2}$ \\ ${ }^{1}$ Universidade Federal do Rio de Janeiro, Escola de Química \\ ${ }^{2}$ Universidade Federal do Rio de Janeiro, Escola de Química, Departamento de Engenharia \\ Química \\ E-mail para contato: jperisse@ufrj.br
}

\begin{abstract}
RESUMO - As mudanças no mercado e no perfil do aluno de engenharia torna necessária a criação de novas metodologias de ensino que confiram ao aluno uma maior flexibilidade e que associem o conhecimento teórico com habilidades práticas exigidas do profissional de engenharia. Pensando nisso, buscou-se desenvolver uma metodologia alternativa na forma de um aplicativo capaz de auxiliar no processo de ensino-aprendizagem através de recursos gráficos, simulações de conceitos teóricos básicos e a correção de uma lista interativa de exercícios.
\end{abstract}

\section{INTRODUÇÃO}

O computador tem sido uma ferramenta essencial para engenheiros, professores e alunos ao longo dos últimos anos. O aumento da importância do computador na engenharia, a evolução rápida de novas aplicações e tecnologias, a atual demanda do mercado industrial por engenheiros com conhecimentos multidisciplinares e o novo perfil do aluno de engenharia criaram a necessidade de uma modernização das metodologias de ensino e de uma maior presença do computador no ensino de engenharia (Iborraet al., 2014).

Kassim e Cadbury (1996) descrevem em seu trabalho que os computadores estão presentes principalmente em cinco tópicos no ensino de engenharia química: planilhas e bancos de dados; modelagem e simulação; controle de processos industriais; linguagens de programação; ferramentas computacionais no processo de ensino-aprendizagem.

O uso de ferramentas computacionais, como planilhas, promove uma maior e mais rápida compreensão do conceito teórico, pois apresenta para o aluno uma aplicação prática e permite que ele interaja com os conceitos, principalmente se associada a uma interface simples (Heyen e Kaliventzeff, 1999).

O trabalho de Kirillovet al. (2015) mostra como as técnicas tradicionais de educação não são suficientes para atender as exigências do atual mercado e como metodologias multidisciplinares e flexíveis contribuem para a criação de um profissional capaz de resolver problemas de maneira inovadora em ambientes de rápida mudança. Fadeeva e Kirillov (2015) ainda afirmam que inovações no ensino, ou seja, novas metodologias e didáticas são responsáveis por aumentar a eficácia do processo educacional.

\section{OBJETIVOS}


Visando estreitar o relacionamento do estudante de engenharia com o computador, buscou-se desenvolver um programa e uma metodologia que permitisse ao usuário simular alguns possíveis cenários de problemas básicos de engenharia e que também fosse uma ferramenta de auxilio no processo de ensino-aprendizagem aliando planilhas e bancos de dados, além de uma interface didática, simples e dinâmica.

\section{METODOLOGIA}

A plataforma escolhida para o desenvolvimento do programa foi Excel/VBA, que não só é familiar a maior parte dos alunos como também pode ter uma ampla aplicação ao longo do curso de engenharia química (Wong e Barford, 2014).

O programa foi dividido em dois projetos complementares. A primeira parte focada na simulação de problemas e conceitos teóricos e a segunda na avaliação e no exercício de conceitos teóricos.

\subsection{Criação de Bancos de Dados}

O simulador: Para ser capaz de simular os conceitos teóricos, o programa necessita de uma grande quantidade de parâmetros. Unidades de engenharia, valores de constantes, fórmulas e até mesmo substâncias químicas foram armazenados em um banco de dados.

$\underline{\mathrm{O} \text { gerador da lista de exercícios: }}$ Para a criação da lista de exercício, foram criados 3 bancos de dados expostos na Tabela 1 .

Tabela 1- Bancos de Dados

\begin{tabular}{cc}
\hline Nome & Função \\
\hline Unidades & $\begin{array}{c}\text { Armazena unidades de engenharia e seus respectivos fatores de conversão para } \\
\text { uma unidade de referência }\end{array}$ \\
Enunciados & $\begin{array}{c}\text { Armazena as perguntas divididas em trechos, alternando em cada coluna } \\
\text { textos fixos e variáveis de valor ou unidade }\end{array}$ \\
Respostas & $\begin{array}{c}\text { Armazena as fórmulas para a resolução de cada pergunta presente no banco de } \\
\text { enunciados }\end{array}$ \\
\hline
\end{tabular}

O banco de dados de Unidades é organizado por dimensões, cada dimensão possui uma matriz $(i, j)$ onde $i$ é a quantidade de unidades dessa dimensão armazenadas no banco de dados e $j$ é 2 . Nas posições $(i, 1)$ estão armazenadas as unidades e nas posições $(i, 2)$ os fatores de conversão da unidade correspondente em relação a uma correspondente em relação a uma unidade referência. Todas as dimensões apresentam um código, listado na tabela 2, pelo qual são identificadas no algoritmo do programa.

O banco de dados de enunciados é uma matriz $(l, m)$, onde $l$ é o número de perguntas cadastradas no banco de dados e $m$ é a quantidade de termos que compõe a pergunta. Esses termos podem ser strings com parte do enunciado ou variáveis que serão calculadas por um dos algoritmos desenvolvidos. Existem duas possibilidades de variáveis nesse banco de dados, a opção de um número variável, que é representada por \#X ou \#Y, e a opção de uma unidade variável, representada pro \#UiD e \#UrD. 
Tabela 2- Lista de Dimensões e Códigos

\begin{tabular}{cc}
\hline Código & Dimensão \\
\hline A & Área \\
E & Energia \\
F & Força \\
G & Diferença de Temperatura \\
H & Tempo \\
L & Comprimento \\
M & Massa \\
N & Quantidade de matéria \\
$\mathbf{P}$ & Pressão \\
$\mathbf{R}$ & Densidade \\
S & Velocidade \\
$\mathbf{T}$ & Temperatura \\
$\mathbf{V}$ & Volume \\
$\mathbf{W}$ & Potência \\
$\mathbf{K}$ & Qualquer um dos temas anteriores \\
\hline
\end{tabular}

O banco de dados de respostas é uma matriz $(l, t)$, onde $l$ é o número de perguntas cadastradas e $\mathrm{t}$ armazena as formulas e os termos utilizados no cálculo das respostas de cada enunciado. Cada linha do banco de dados de enunciados corresponde a uma linha no banco de dados de resposta. O banco de dados de respostas ainda recebe os valores dos fatores de conversão e das variáveis \#X ou \#Y gerados pelos algoritmos desenvolvidos e utiliza esses valores para produzir a resposta da pergunta gerada.

\subsection{Desenvolvimento dos Algoritmos}

Ambos os projetos requerem a criação de algoritmos que conferem uma maior flexibilidade ao programa. O algoritmo desenvolvido para o simulador é capaz de simular problemas e gerar resultados com parâmetros default ou personalizados.

Para o gerador de lista de exercícios foram gerados 3 algoritmos. $\mathrm{O}$ algoritmo principal Pergunta foi desenvolvido para formular o enunciado, através do somatório de vários termos (partes do enunciado, números aleatórios e unidades aleatórias), como representado na equação 1.

Pergunta $=\sum P_{l, m}$

$\mathrm{O}$ segundo algoritmo $(\mathrm{N})$, exposto na equação 2 , gera um número aleatório a partir de limites superiores (S) e inferiores (I) definidos e com um número de casas decimais (R). Esta função é utilizada no algoritmo Pergunta para definir qual linha $l$ da matriz do banco de dados de Enunciados que vai ser montada pelo algoritmo Pergunta. Neste caso, o limite inferior (I) é a linha da primeira pergunta e o limite superior (S) é o número de perguntas cadastradas. Além disso, é utilizada para gerar números aleatórios que servem de argumento no enunciado.

$$
N(I, S, R)=x €[I, S]
$$


O terceiro algoritmo (U), representado na equação 3, é utilizado para selecionar uma unidade aleatória presente no banco de dados de Unidades. Tem como parâmetros o código da dimensão (D), definido em um dos termos da função Pergunta e um valor aleatório gerado pelo algoritmo $\mathrm{N}$, que define a unidade selecionada. Neste caso, os limites superiores (S) e inferiores (I) do algoritmo $\mathrm{N}$ são determinados pelo parâmetro $\mathrm{D}$, ou seja, cada dimensão possui limites diferentes. O resultado do terceiro algoritmo é um vetor, onde o elemento U1 é um string correspondente a unidade selecionada e é utilizado na função Pergunta. O elemento U2 é um número correspondente ao fator de conversão dessa unidade para a unidade de referência dessa dimensão e é utilizado para calcular a resposta.

$$
U_{N, k}(D, N)=\left[\begin{array}{ll}
U_{1} & U_{2}
\end{array}\right]
$$

\section{RESULTADOS}

Aplicando os conceitos teóricos básicos de engenharia química ao simulador e ao aplicativo gerados de lista de exercícios foram gerados dois programas utilizando os bancos de dados criados e os algoritmos desenvolvidos. Para facilitar o uso dos programas foram criadas interfaces gráficas que facilitam a utilização da aplicação pelo usuário.

\subsection{O Simulador}

Foi criado um programa capaz de apresentar resultados numéricos de conceitos teóricos básicos como densidade, pressão e capacidade calorífica a partir de dados de entrada que podiam ser adicionados pelo usuário de maneira direta ou a partir da interação com os recursos visuais ou importados do banco de dados do próprio programa. Todos os resultados vinham acompanhados de uma representação gráfica dinâmica e interativa, no formato de tanques ou termômetros.

O desenvolvimento do simulador envolveu tanto um código de programação quanto a criação de uma interface simples e dinâmica. A interface do programa conforme mostra a Figura 1consiste em um menu principal, um manual de instruções, 2 módulos de simulação e uma página de configurações.

Figura 1 - Interface gráfica do simulador

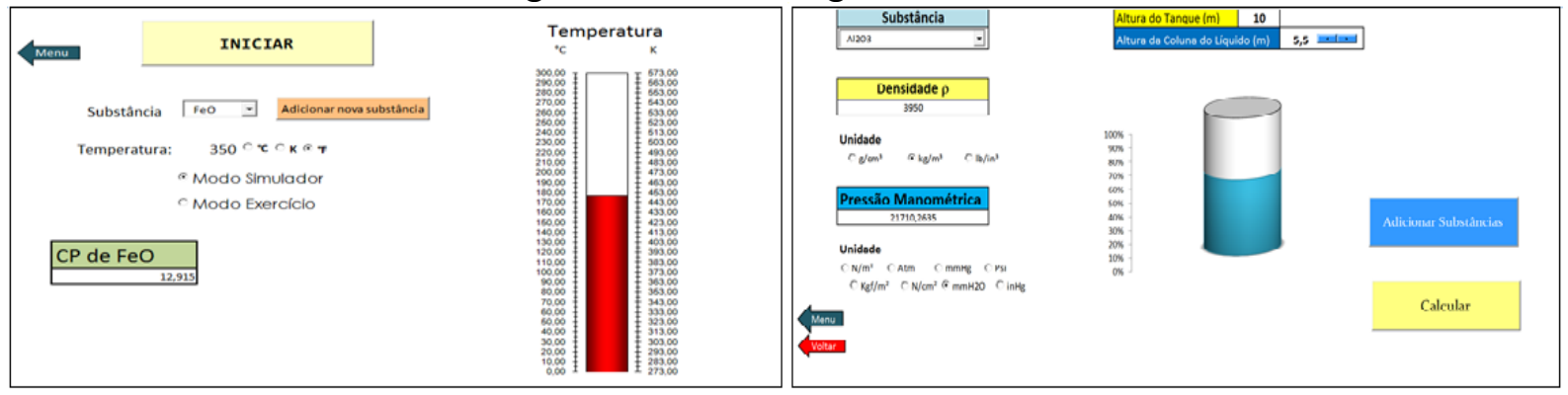

\subsection{O Gerador de Lista de Exercícios}


As listas de exercícios são criadas a partir da repetição dos algoritmos. As perguntas geradas a partir da junção de trechos fixos vindo do banco de Enunciados e trechos variáveis gerados aleatoriamente pelo próprio programa. Todos os valores variáveis gerados pelo programa são armazenados no banco de dados de Respostas e inseridos na fórmula referente a linha do enunciado selecionada para gerar a resposta da pergunta criada. Esse procedimento é repetido até gerar o número de perguntas definido para formar a lista de exercício. Essa quantidade pode ser alterada, gerando listas de diferentes tamanhos.

Gerada a lista de exercício, o programa, então, apresenta a lista para o usuário através de uma interface gráfica, apresentada na figura 2. Após, a resolução das questões, o usuário pode escolher entre armazenar a lista num arquivo ou corrigir as questões na própria interface. $\mathrm{O}$ arquivo gerado contém o nome do aluno e seu registro, todas as perguntas da lista de exercícios feita, a resposta correta calculada pelo programa de cada pergunta, as respostas do usuário e o tempo de duração do exercício. Já, a correção na tela, apresenta o tempo de duração do exercício e a representação gráfica dos erros e acertos.

Figura 2 - Parte da lista de exercícios gerada apresentando uma pergunta, a calculadora e os botões.

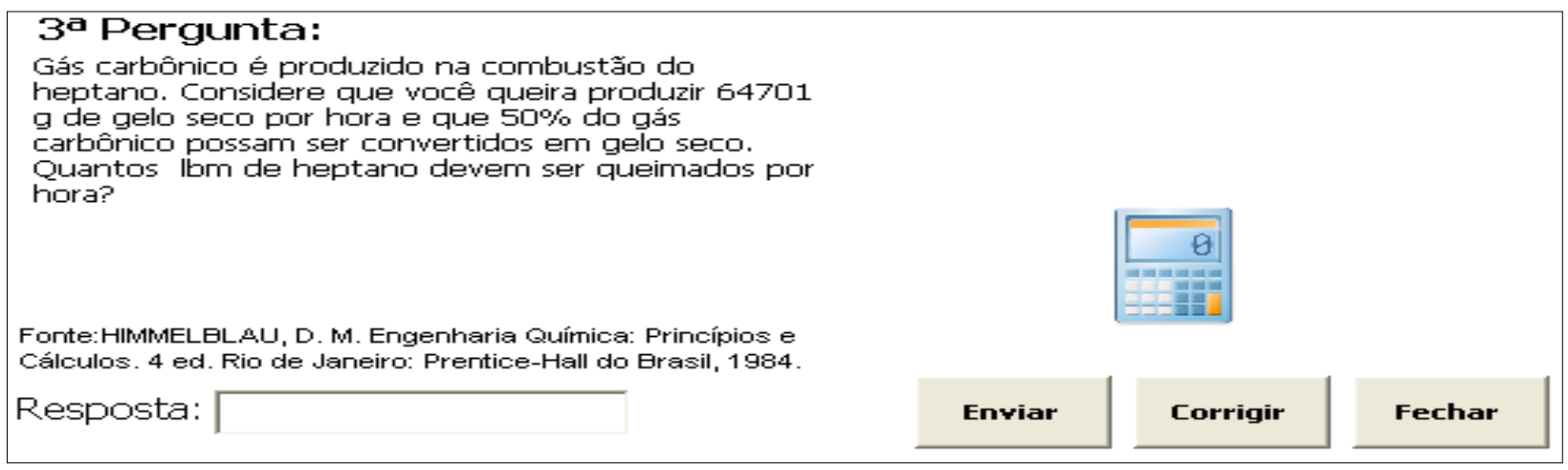

O banco de Enunciados possui, atualmente, 50 modelos de perguntas sobre conceitos básicos de engenharia, de diferentes fontes, cadastradas. O banco de dados de Unidades apresenta 14 dimensões básicas e combinadas cadastradas, conforme apresentado anteriormente na tabela 2.

\section{CONCLUSÃO}

Foi possível com esse trabalho desenvolver a metodologia de ensino proposta e aplicála a uma ferramenta computacional, utilizando recursos gráficos e os algoritmos desenvolvidos. O aplicativo criado pode ser facilmente utilizado como ferramenta complementar ao ensino de uma disciplina, além de, poder ser facilmente adaptado aos mais diversos assuntos, cursos e matérias, devido a sua natureza flexível.

O programa foi aplicado aos conceitos básicos de engenharia química e foi utilizado como ferramenta complementar em uma disciplina de $1^{\circ}$ período dos cursos da Escola de Química da Universidade Federal do Rio de Janeiro. 
O atual banco de dados de Unidades apresenta dimensões e unidades cadastradas que contempla uma gama de disciplinas. Logo, a adaptação da ferramenta geradora da lista de exercícios para outros assuntos necessita apenas que sejam adicionados novas perguntas ao banco de dados de Enunciados.

A metodologia criada conseguiu atingir todos os itens exigidos, desde as suas interfaces gráficas até a sua flexibilidade.

\section{REFERENCIAS}

FADEEVA, V. N.; KIRILLOV, N. P. The Issues of Development of a Creative Professional.Elsevier, Procedia - Social and Behavioral Sciences, v. 166, p. 333-338, 2015.

HEYEN, G.; KALITVENTZEFF, B. Spreadsheetbased teaching aids in chemical engineering education.Elsevier, Computers and Chemical Engineering Supplement, p. S629-S632, 1999.

IBORRA, M.; RAMÍREZ, E.; TEJERO, J.; BRINGUÉ, R.; FITÉ, C.; CUNILL, F. Revamping of teaching-learning methodologies inlaboratory subjects of the Chemical Engineeringundergraduate degree of the University ofBarcelona for their adjustment to the Bologna process.Elsevier B.V.,The Institution of Chemical Engineers, 2014.

KASSIM, H. O.;CADBURY, R. G.The Place of the Computer in Chemical Engineering Education.Elsevier, Computers and Chemical Engineering Supplement,v.20, p. S1341S1346, 1996.

KIRILLOV, N. P.; LEONTYEVA, E. G.; MOISEENKO, Y.A. Creativity in engineering education.Elsevier, Procedia - Social and Behavioral Sciences, v. 166, p. 360-363, 2015.

WONG, K. W. W.; BARFORD, J. P. Teaching Excel VBA as a problem solving tool for chemical engineering core courses.Elsevier B.V.,The Institution of Chemical Engineers, 2010. 